\title{
Clinical characteristics of inflammatory bowel disease patients with immunoglobulin A nephropathy
}

\author{
Ryohei Hayashi ${ }^{1}$, Yoshitaka Ueno ${ }^{1}$, Shinji Tanaka ${ }^{1}$, Kana Onishi $^{2}$, Takeshi Takasago ${ }^{2}$, Masaki Wakai $^{2}$, Toshikatsu Naito ${ }^{2}$, \\ Kensuke Sasaki ${ }^{3}$, Shigehiro Doi ${ }^{3}$, Takao Masaki ${ }^{3}$, Kazuaki Chayama ${ }^{2,4}$ \\ Departments of ${ }^{1}$ Endoscopy, ${ }^{2}$ Gastroenterology and Metabolism, and ${ }^{3}$ Nephrology, Hiroshima University Hospital, Hiroshima; ${ }^{4}$ Institute of \\ Physical and Chemical Research (RIKEN) Center for Integrative Medical Sciences, Yokohama, Japan
}

Background/Aims: Inflammatory bowel disease (IBD) is a chronic inflammation of the gastrointestinal tract. Some patients with this condition have been reported to present with immunoglobulin A nephropathy (IgAN), a renal complication that can cause end-stage renal failure, but the frequency of this comorbidity has not been described. Thus, the aim of this study was to investigate the frequency of IgAN in patients with IBD. Methods: This study included 620 patients with IBD (338 with ulcerative colitis [UC] and 282 with Crohn's disease [CD]) from the Hiroshima University Hospital outpatient department. IgAN cases were identified from medical interviews, blood examinations (serum immunoglobulin A), and urinalyses (occult blood, proteinuria). Definitive IgAN cases were diagnosed by renal biopsies, while those detected through the clinical course and test results, but not clinically recommended for renal biopsy, were defined as suspected IgAN. Results: We analyzed 427 cases meeting the inclusion criteria ( 220 with UC and 207 with CD). The incidence of IgAN across all patients with IBD was $3.0 \%$. The frequency of IgAN was significantly higher in patients with CD $(11 / 207,5.3 \%)$ than in those with UC $(2 / 220,0.9 \%)(P<0.01)$. Moreover, a significant correlation was found between CD patients with ileostomy or colostomy and a diagnosis of IgAN. Conclusions: Patients with IBD present a high incidence of IgAN, especially those with CD who have undergone ileostomy or colostomy. (Intest Res 2021;19:430-437)

Key Words: Inflammatory bowel disease; Crohn disease; Colitis, ulcerative; Immunoglobulin A nephropathy; Extraintestinal manifestation

\section{INTRODUCTION}

Inflammatory bowel disease (IBD) is a chronic inflammation of the gastrointestinal tract that results in mucosal ulceration and tissue damage. Two of the major clinically defined forms of IBD are ulcerative colitis (UC) and Crohn's disease (CD). Although the etiology of IBD has not been fully elucidated, the disease process includes multiple factors, such as genetic background, mucosal barrier dysfunction, intestinal microbio-

Received June 4, 2020. Revised July 3, 2020. Accepted July 8, 2020. Correspondence to Yoshitaka Ueno, Department of Endoscopy, Hiroshima University Hospital, 1-2-3 Kasumi, Minami-ku, Hiroshima 734-8551, Japan. Tel: +81-82-257-5191, Fax: +81-82-257-5194,

E-mail:yueno@hiroshima-u.ac.jp ta, and environmental factors. ${ }^{1-3}$ Numerous extraintestinal manifestations occur in IBD, including lesions of the skin (erythema nodosum and pyoderma gangrenosum $)^{4}$ and of the joints (peripheral arthritis, sacroiliitis, and ankylosing spondylitis), ${ }^{5,6}$ hepatobiliary complications (gallstones and primary sclerosing cholangitis), ${ }^{7,8}$ and renal complications. ${ }^{9}$ Immunoglobulin A nephropathy (IgAN) is one of the rare renal complications associated with IBD..$^{10-13}$

IgAN is one of the most predominant forms of glomerulonephritis, ultimately leading to end-stage renal failure in a high proportion of patients (approximately $30 \%-40 \%) .^{14}$ The International Kidney Biopsy Survey on glomerular disease frequencies, which consisted of 42,603 renal biopsies spanning 4 continents, diagnosed IgAN in $22.1 \%$ of all glomerular disease 
in Europe and 39.5\% in Asia. ${ }^{15}$ Although most cases of IgAN are considered as a primary disease, some cases occur as secondary IgAN associated with liver diseases (alcoholic, primary biliary or cryptogenic cirrhosis, and hepatitis B), skin diseases (dermatitis herpetiformis and psoriasis), neoplasia, infection (human immunodeficiency virus and leprosy), lung disease (sarcoidosis, idiopathic pulmonary hemosiderosis, cystic fibrosis, and bronchiolitis obliterans), and IBD (UC and CD). ${ }^{10}$ Despite some cases of IgAN being reported to occur together with IBD, the frequency of co-occurrence has not been investigated. Therefore, the aim of the present study was to investigate the frequency of IgAN in patients with IBD.

\section{METHODS}

\section{Patients}

We performed a retrospective cohort study to evaluate the frequency of IgAN in patients with IBD and to assess risk factors of IBD. This study included 338 patients with UC and 282 with CD (620 in total) who consecutively visited the Hiroshima University Hospital outpatient department between November 2016 and January 2017. Because 193 patients did not give consent, we analyzed a total of 427 patients (220 UC and 207 $\mathrm{CD}$ ). The diagnosis of UC or CD was made according to the clinical symptoms, endoscopic findings, and the presence of histological features compatible with these diseases.

Montreal classification was used to define the extent and location of intestinal lesions, with UC patients defined as ulcerative proctitis (E1), left-sided UC (E2), or extensive UC (E3), and CD patients classified as ileal (L1), colonic (L2), or ileocolonic (L3) ${ }^{16}$ Clinical activity was evaluated using the Rachmilewitz index (clinical activity index) in UC patients, ${ }^{17}$ and Crohn's Disease Activity Index in CD patients. ${ }^{18}$

\section{Screening of IgAN}

IgAN screening consisted of a medical interview, blood examination, and urinalysis. For the blood examination we assessed serum IgA, creatinine, C-reactive protein, hemoglobin and sedimentation tests. Urinalysis measured occult blood, microscopic hematuria, and proteinuria. We consulted the following cases to the nephrologists: (1) serum IgA $\geq 315 \mathrm{mg} / \mathrm{dL}$ and persistent microscopic hematuria, or (2) proteinuria and persistent microscopic hematuria. The cases of definitive diagnosis by renal biopsy were defined as "definite IgAN (dIgAN)," and the cases of suspected IgAN based on the clinical course and test results, but not clinically recommended for renal bi- opsy, were defined as "suspected IgAN (sIgAN)." Patients who had been diagnosed with IgAN in past renal biopsies were also classed as dIgAN if the findings were confirmed by medical interview and medical records. Similarly, those who had previously been suspected as IgAN without renal biopsy were also defined as sIgAN.

\section{Statistical Analyses}

Statistical analyses were performed using JMP Pro 14.2.0 (SAS Institute, Cary, NC, USA) and Prism 5.0 (GraphPad, San Diego, CA, USA). Student t-test with Welch correction for unpaired data or the Fisher exact test was used to compare data between the 2 groups. Multivariate analyses were conducted by logistic regression analysis, and $P<0.05$ were considered statistically significant.

\section{Ethical Considerations}

Our study was approved by the Hiroshima University Hospital Institutional Review Board (IRB No. E-798). Our protocols conformed to the ethical standards of the responsible committees on human experimentation (institutional and national) and with the Helsinki Declaration of 1964 and later versions. Informed consent was obtained from all patients included in this report.

\section{RESULTS}

\section{Clinical Characteristics of Patients and Screening Results}

We conducted IgAN screening on 427 patients, including 220 UC patients and 207 CD patients. The background characteristics for all patients are shown in Table 1. As a result of medical interviews, 5 patients were classified as dIgAN due to previous renal biopsies, and 2 were classified as sIgAN as they had previously been suspected of having IgAN but renal biopsy had not been carried out. The full screening results are shown in Fig. 1.

Blood and urinary examinations showed no significant differences between UC and CD patients in serum creatinine, Creactive protein levels, or the frequency of urinary occult blood. However, serum IgA levels and the frequency of proteinuria were significantly higher in CD patients (Table 2). These abnormal blood and urinalysis findings were seen in 29 patients (9 UC and $20 \mathrm{CD}$ ). Among them, 4 patients were diagnosed with diseases other than renal disease: 3 with urinary lithiasis and one with prostatic cancer. Two patients were ex- 
Table 1. Clinical Characteristics of Patients at Baseline

\begin{tabular}{|c|c|c|}
\hline Variable & $U C(n=220)$ & $C D(n=207)$ \\
\hline Male sex & $122(55)$ & $151(73)$ \\
\hline Age (yr) & $49.6 \pm 15.6$ & $41.3 \pm 3.0$ \\
\hline Disease duration (yr) & $11.2 \pm 8.9$ & $15.4 \pm 10.6$ \\
\hline \multicolumn{3}{|l|}{ Montreal classification } \\
\hline \multicolumn{3}{|l|}{ Extent (UC) } \\
\hline E1 & $49(22)$ & \\
\hline E2 & $52(24)$ & \\
\hline E3 & $119(54)$ & \\
\hline \multicolumn{3}{|l|}{ Location (CD) } \\
\hline L1 & & $61(29)$ \\
\hline L2 & & $12(6)$ \\
\hline L3 & & $134(65)$ \\
\hline \multicolumn{3}{|l|}{ Clinical score } \\
\hline CAI (UC) & $1(0-12)$ & - \\
\hline CDAI (CD) & - & $204(10-394)$ \\
\hline \multicolumn{3}{|l|}{ Current/past therapies } \\
\hline Mesalamine & $216(98)$ & 193 (93) \\
\hline Corticosteroids & $135(61)$ & $110(53)$ \\
\hline Immunomodulators & $103(47)$ & $83(40)$ \\
\hline Calcineurin inhibitors & $29(13)$ & 0 \\
\hline Biologics & $41(19)$ & $125(60)$ \\
\hline Cytapheresis & $54(25)$ & $2(1)$ \\
\hline
\end{tabular}

Values are presented as number (\%), mean \pm standard deviation, or median (range).

UC, ulcerative colitis; CD, Crohn's disease; E1, ulcerative proctitis; E2, left-sided UC; E3, extensive UC; L1, ileal; L2, colonic; L3, ileocolonic; CAI, clinical activity index; CDAl, Crohn's Disease Activity Index.

cluded because they discontinued their hospital visit. The remaining 23 patients (5 UC and $18 \mathrm{CD}$ ) were consulted by nephrologists. Four cases were subjected to renal biopsy, and 2 were diagnosed with dIgAN. The remaining 2 were diagnosed with other renal diseases by renal biopsy: one case was diagnosed with minor glomerular abnormalities, and one case was diagnosed with renal amyloidosis. An additional 4 patients were diagnosed with sIgAN but were unable to undergo renal biopsy because of renal cortical thinning, high activity of IBD or patient rejection (Fig. 1).

Finally, 6 cases (1.4\%) were diagnosed with sIgAN, and 7 cases $(1.6 \%)$ were diagnosed with dIgAN. The incidence of sIgAN and dIgAN was significantly higher in CD patients than in those with UC (CD 5.3\% vs. UC $0.9 \%, P<0.01$ ) (Table 2).

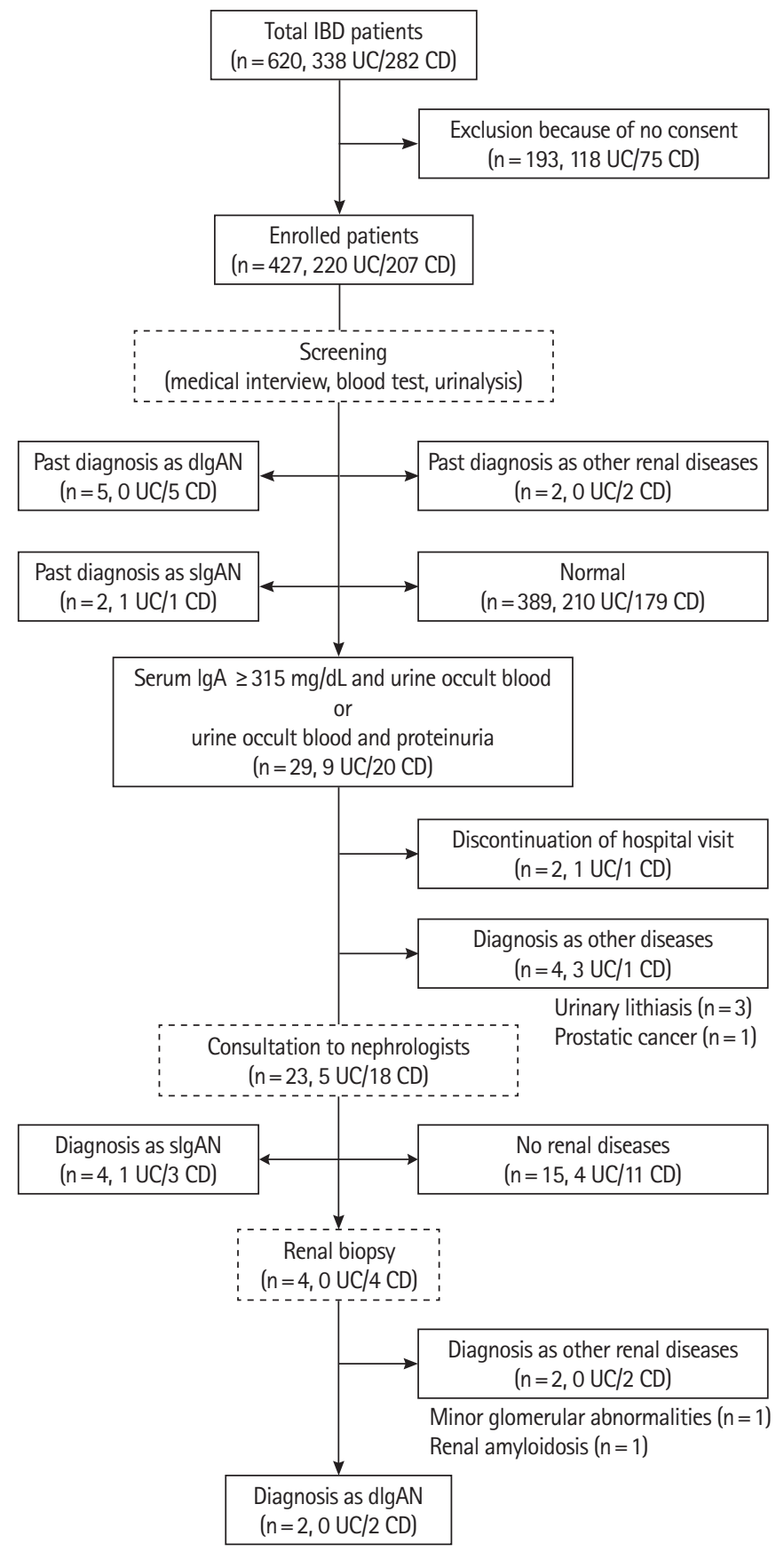

Fig. 1. Flow diagram of patients enrolled in this study. IBD, inflammatory bowel disease; UC, ulcerative colitis; $C D$, Crohn's disease; IgA, immunogloburin $A_{\text {; }} \lg A N$, IgA nephropathy; slgAN, suspected IgAN; dlgAN, definite IgAN.

\section{Clinical Characteristics of IgAN Cases}

IBD patients diagnosed with IgAN experienced a significantly longer duration of disease than did patients without IgAN, but no differences in sex, age or current/past therapies were evident between the groups (Table 3). 
Table 2. Results of IgAN Patients Screening

\begin{tabular}{lccc}
\hline Result & UC $(\mathrm{n}=220)$ & CD $(\mathrm{n}=207)$ & $P$-value \\
\hline Blood exam & & & \\
$\operatorname{lgA}(\mathrm{mg} / \mathrm{dL})$ & $238.7 \pm 98.4$ & $313.5 \pm 137.2$ & $<0.001$ \\
$\mathrm{Cr}(\mathrm{mg} / \mathrm{dL})$ & $0.7 \pm 0.2$ & $1.1 \pm 3.6$ & $\mathrm{NS}$ \\
CRP $(\mathrm{mg} / \mathrm{dL})$ & $0.3 \pm 1.6$ & $0.5 \pm 1.1$ & $\mathrm{NS}$ \\
Urinary exam & & & \\
Occult blood & $41(18.6)$ & $33(15.9)$ & $\mathrm{NS}$ \\
Proteinuria & $7(3.2)$ & $22(10.6)$ & $<0.010$ \\
IgA nephropathy & & & \\
slgAN & $2(0.9)$ & $4(1.9)$ & $\mathrm{NS}$ \\
dlgAN & 0 & $7(3.3)$ & $<0.010$ \\
Total (slgAN+dlgAN) & $2(0.9)$ & $11(5.3)$ & $<0.010$ \\
\hline
\end{tabular}

Values are presented as mean \pm standard deviation or number (\%).

anot significant (NS), $P>0.05$.

UC, ulcerative colitis; $C D$, Crohn's disease; IgA, immunogloburin $A ; C r$, creatinine; CRP, C-reactive protein; IgAN, IgA nephropathy; slgAN, suspected IgAN; dlgAN, definite lgAN.

Table 4. Clinical Characteristics of CD Patients at Time of IgAN Diagnosis

\begin{tabular}{lcccc}
\hline Variable & $\begin{array}{c}\text { Without } \\
\text { IgAN } \\
(\mathrm{n}=195)\end{array}$ & $\begin{array}{c}\text { With } \\
\text { slgAN } \\
(\mathrm{n}=4)\end{array}$ & $\begin{array}{c}\text { With } \\
\text { dlgAN } \\
(\mathrm{n}=7)\end{array}$ & $\begin{array}{c}P \text { - } \\
\text { value }^{\mathrm{a}}\end{array}$ \\
\hline Male sex & $142(73)$ & $4(100)$ & $5(71)$ & NS \\
Age (yr) & $41.2 \pm 13.0$ & $49.8 \pm 13.5$ & $38.4 \pm 16.1$ & NS \\
Disease duration (yr) & $15.2 \pm 10.7$ & $21.0 \pm 9.5$ & $16.4 \pm 10.3$ & NS \\
Location & & & & NS \\
L1 & $61(31)$ & 0 & 0 & \\
L2 & $11(6)$ & 0 & $1(14)$ & \\
L3 & $123(63)$ & $4(100)$ & $6(86)$ & \\
Current/past therapies & & & & \\
Mesalamine & $181(93)$ & $4(100)$ & $6(86)$ & NS \\
Corticosteroids & $104(53)$ & $3(75)$ & $2(29)$ & NS \\
Immunomodulators & $79(41)$ & $2(50)$ & $1(14)$ & NS \\
Biologics & $117(60)$ & $3(75)$ & $3(43)$ & NS \\
Cytapheresis & $2(1)$ & 0 & 0 & NS \\
Abdominal surgery & $118(61)$ & $4(100)$ & $4(57)$ & NS \\
Ileostomy/colostomy & $20(10)$ & $3(75)$ & $3(43)$ & $<0.001$ \\
\hline
\end{tabular}

Values are presented as number (\%) or mean \pm standard deviation.

'Without IgAN versus with slgAN, with dlgAN (not significant [NS], $P>0.05)$.

$C D$, Crohn's disease; IgAN, immunoglobulin A nephropathy; slgAN, suspected IgAN; dlgAN, definite IgAN; L1, ileal; L2, colonic; L3, ileocolonic.

Since most patients diagnosed with sIgAN or dIgAN experienced CD (84.6\%, 11/13 cases), we next specifically examined the characteristics of the $\mathrm{CD}$ cohort of patients. There were no
Table 3. Clinical Characteristics of IBD Patients with/without IgAN at Baseline

\begin{tabular}{|c|c|c|c|c|}
\hline Variable & $\begin{array}{l}\text { Without } \\
\lg A N \\
(n=412)\end{array}$ & $\begin{array}{l}\text { With } \\
\text { slgAN } \\
(n=6)\end{array}$ & $\begin{array}{l}\text { With } \\
\text { dlgAN } \\
(n=7)\end{array}$ & $P$-value ${ }^{a}$ \\
\hline Male sex & $262(64)$ & $6(100)$ & $5(71)$ & NS \\
\hline Age (yr) & $45.5 \pm 14.8$ & $51.0 \pm 15.7$ & $44.7 \pm 13.2$ & NS \\
\hline Diagnoses & & & & $<0.010$ \\
\hline UC & 217 (53) & $2(33)$ & 0 & \\
\hline$C D$ & $195(47)$ & $4(67)$ & $7(100)$ & \\
\hline Disease duration (yr) & $13.0 \pm 9.9$ & $22.2 \pm 10.7$ & $23.0 \pm 8.5$ & $<0.010$ \\
\hline \multicolumn{5}{|l|}{ Blood exam } \\
\hline $\lg \mathrm{A}(\mathrm{mg} / \mathrm{dL})$ & $267 \pm 112$ & $398 \pm 148$ & $601 \pm 243$ & $<0.010$ \\
\hline $\mathrm{Cr}(\mathrm{mg} / \mathrm{dL})$ & $0.9 \pm 2.5$ & $1.4 \pm 1.0$ & $1.2 \pm 0.5$ & NS \\
\hline CRP (mg/dL) & $0.4 \pm 1.4$ & $0.5 \pm 0.8$ & $0.5 \pm 0.5$ & NS \\
\hline \multicolumn{5}{|l|}{ Urinary exam } \\
\hline Occult blood & $60(15)$ & $6(100)$ & $6(86)$ & $<0.001$ \\
\hline Proteinuria & $19(3)$ & $4(67)$ & $5(71)$ & $<0.001$ \\
\hline Current/past therapies & & & & NS \\
\hline Mesalamine & 394 (96) & $7(100)$ & $7(100)$ & \\
\hline Corticosteroids & 237 (58) & $4(67)$ & $3(43)$ & \\
\hline Immunomodulators & $181(44)$ & $2(33)$ & $2(29)$ & \\
\hline Calcineurin inhibitors & $29(7)$ & 0 & 0 & \\
\hline Biologics & $158(38)$ & $3(50)$ & $4(57)$ & \\
\hline Cytapheresis & $55(13)$ & 0 & 0 & \\
\hline
\end{tabular}

Values are presented as number (\%) or mean \pm standard deviation.

Without IgAN versus with slgAN, with dlgAN (not significant [NS], $P>0.05$ ).

$I B D$, inflammatory bowel disease; IgA, immunoglobulin $A$; $\lg A N, \lg A$ nephropathy; slgAN, suspected IgAN; dlgAN, definite IgAN; UC, ulcerative colitis; CD, Crohn's disease; $\mathrm{Cr}$, creatinine; $\mathrm{CRP}, \mathrm{C}$-reactive protein.

significant differences in sex, age, disease duration, disease location or current/past therapies (including abdominal surgery) between CD patients with IgAN and those without IgAN. However, whether or not ileostomy/colostomy was performed showed a significant difference between the 2 groups at the time of IgAN diagnosis (Table 4). Multivariate analysis using logistic regression analysis showed that performing ileostomy/ colostomy was significantly associated with IgAN (Table 5).

The clinical characteristics of all patients diagnosed with IgAN are shown Table 6. Only one patient was simultaneously diagnosed with IBD and IgAN, while all other cases received a diagnosis of IBD prior to undergoing diagnosis of IgAN. All ileostomies/colostomies in CD patients were permanent. 
Table 5. Factors Associated with IgAN in CD Patients

\begin{tabular}{lcc}
\hline Patients $(\mathrm{n}=206)$ & Multivariate OR (95\% CI) & $P$-value \\
\hline Male sex & $3.08(0.43-22.10)$ & 0.262 \\
Age & $1.70(0.40-9.11)$ & 0.495 \\
Disease duration & $0.59(0.11-2.50)$ & 0.489 \\
Small-intestinal lesion & $0.95(0.78-11.55)$ & 0.966 \\
Current/past therapies & & \\
Mesalamine & $0.75(0.06-9.71)$ & 0.825 \\
Corticosteroids & $1.36(0.27-6.80)$ & 0.711 \\
Immunomodulators & $0.42(0.08-2.26)$ & 0.312 \\
Biologics & $0.52(0.11-2.59)$ & 0.427 \\
Abdominal surgery & $1.30(0.17-9.77)$ & 0.801 \\
Ileostomy/colostomy & $29.60(4.65-188.31)$ & $<0.001^{\mathrm{a}}$ \\
\hline
\end{tabular}

astatistically significant.

IgAN, immunoglobulin A nephropathy; CD, Crohn's disease; OR, odds ratio; $\mathrm{Cl}$, confidence interval.

\section{DISCUSSION}

To our knowledge, this is the first report screening and investigating the incidence of IgAN in patients with IBD. The overall incidence of IgAN in Japan is considered as 4.5 cases/year per $100,000 .{ }^{19}$ A systematic review of 40 studies from Europe, North and South America, Canada, Australasia and the Middle East found an incidence of IgAN in adults of 2.5 cases/year per $100,000 .{ }^{20}$ In the present study, among 427 patients with IBD who visited our hospital, 3.0\% had IgAN comorbidity. Although this cannot simply be compared with the overall incidence rate, we propose that our study reveals a high rate of IgAN in patients with IBD. IgAN has previously been reported as the most frequent kidney biopsy diagnosis in IBD and has a significantly higher diagnostic prevalence than all non-IBD kidney biopsy diagnoses. ${ }^{21}$ Our study provides further support to these findings. However, a large-scale, multi-center survey will be required to provide more accurate information on the incidence rate of IgAN in patients with IBD.

There are likely to be some cases in which the deterioration of renal function progresses in latency unless proper screening is performed in IBD cases. Indeed, our cohort included one patient in whom renal atrophy had already progressed when IgAN was suspected and renal biopsy could not be performed. Accordingly, we suggest that gastroenterologists need to consider that kidney disease is one of the complications of IBD and therefore should evaluate daily medical treatments with this in mind.
Why do IBD and IgAN co-occur? A major reason may be that IgA, considered to play a crucial role in IgAN, is prevalent in mucosal secretions. ${ }^{22}$ Tonsil and upper respiratory mucosa have attracted attention as sources of IgA immunoconjugates in IgAN, but intestinal mucosa has also attracted attention in recent years. ${ }^{23}$ Mucosa-associated lymphoid tissue is present in induction areas of organized lymphoepithelial tissue, including nasopharynx-associated lymphoid tissue, bronchusassociated lymphoid tissue, and gut-associated lymphoid tissue. ${ }^{24}$ In these areas, mucosal IgA production is induced by both $\mathrm{T}$ cell-independent and $\mathrm{T}$ cell-dependent mechanisms. ${ }^{25}$ Increased small intestinal mucosal permeability has also been demonstrated in IgAN, and the intestinal mucosa is one possible source of IgA in IgAN. ${ }^{26}$ Using a mouse strain with high serum levels of IgA, Kamata et al. ${ }^{27}$ demonstrated that intestinal IgA-producing plasma cells contribute to hyper serum IgA levels and cause IgA deposition in the kidney.

Additionally, recent genome-wide association studies have identified multiple susceptibility loci for IgAN. Interestingly, included in these genomic regions were TNFSF13 and $D E F A .{ }^{28}$ TNFSF13 genes encode APRIL, a tumor necrosis factor ligand involved in $\mathrm{B}$ cell development, the response to mucosal antigens, and the production of IgA in gut-associated lymphoid tissue. DEFA genes encode $\alpha$-defensins, which are natural antimicrobials expressed by small intestinal Paneth cells. Recent reports have found decreased levels of $\alpha$-defensin in patients with $\mathrm{CD}^{29,30}$ Although the exact cause of the high incidence of IgAN in patients with IBD is unclear, these previous reports indicate that intestinal IgA might be affected. It is unclear why performing ileostomy/colostomy was associated with IgAN, but it might involve the intestinal immune response to the normal bacterial flora of the skin.

Our study was not without limitations. Firstly, we assessed a limited number of patients from a single center. Due to a short screening period (3 months), most patients had only 1 or 2 opportunities to undergo blood and urinary examinations. As some patients refused to undergo these examinations, approximately $70 \%$ of outpatients remained eligible for enrollment in this study. Secondly, as clinical activity changes over time and because we included patients diagnosed with IgAN in the past, we were unable to investigate the relationship between the clinical activity of IBD and the incidence of IgAN.

In conclusion, patients with IBD, especially those with CD, present with a high incidence of IgAN. Moreover, performing ileostomy/colostomy may be a risk factor for IgAN. Therefore, attention needs to be paid when performing urinalysis to pre- 


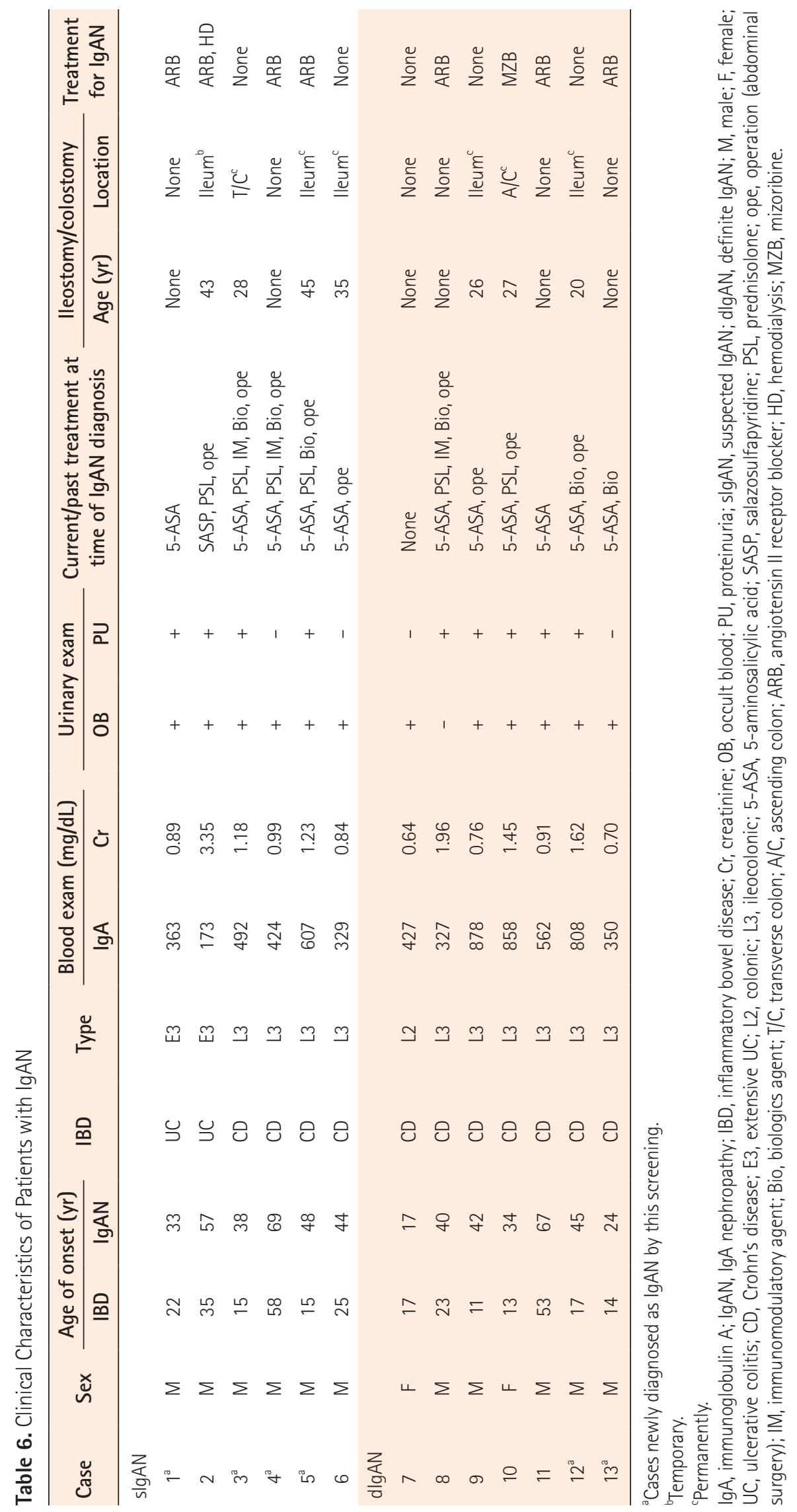


vent the progression of IgAN to renal failure being overlooked in patients with IBD.

\section{ADDITIONAL INFORMATION}

\section{Funding Source}

The authors received no financial support for the research, authorship, and/or publication of this article.

\section{Conflict of Interest}

No potential conflict of interest relevant to this article was reported.

\section{Author Contribution}

Conceptualization: Hayashi R, Ueno Y, Tanaka S, Sasaki K, Chayama K. Data curation: Hayashi R, Onishi K, Takasago T, Wakai M, Naito T. Formal analysis: Hayashi R. Investigation: Hayashi R, Ueno Y, Tanaka S, Onishi K, Takasago T, Wakai M, Naito T, Sasaki K, Doi S, Masaki T. Methodology: Hayashi R, Ueno Y, Tanaka S, Sasaki K, Masaki T, Chayama K. Project administration: Ueno Y, Tanaka S, Masaki T, Chayama K. Resources: Hayashi R, Ueno Y, Tanaka S, Onishi K, Takasago T, Wakai M, Naito T, Sasaki K, Doi S, Masaki T. Supervision: Ueno Y, Tanaka S, Masaki T, Chayama K. Validation: Ueno Y. Visualization: Hayashi R, Ueno Y, Tanaka S. Writing - original draft: Hayashi R, Ueno Y. Writing - review \& editing: all authors. Approval of final manuscript: all authors.

\section{ORCID}

Hayashi R

Ueno $\mathrm{Y}$

Tanaka S

Onishi K

Takasago T

Wakai M

Naito T

Sasaki K

Doi S

Masaki T

Chayama K

\section{REFERENCES}

1. Kaser A, Zeissig S, Blumberg RS. Inflammatory bowel disease. Annu Rev Immunol 2010;28:573-621.

2. Danese S, Fiocchi C. Ulcerative colitis. N Engl J Med 2011;
365:1713-1725.

3. Maloy KJ, Powrie F. Intestinal homeostasis and its breakdown in inflammatory bowel disease. Nature 2011;474:298-306.

4. Gregory B, Ho VC. Cutaneous manifestations of gastrointestinal disorders. Part II. J Am Acad Dermatol 1992;26:371-383.

5. Gravallese EM, Kantrowitz FG. Arthritic manifestations of inflammatory bowel disease. Am J Gastroenterol 1988;83:703709 .

6. Orchard TR, Thiyagaraja S, Welsh KI, Wordsworth BP, Hill Gaston JS, Jewell DP. Clinical phenotype is related to HLA genotype in the peripheral arthropathies of inflammatory bowel disease. Gastroenterology 2000;118:274-278.

7. Ardizzone S, Puttini PS, Cassinotti A, Porro GB. Extraintestinal manifestations of inflammatory bowel disease. Dig Liver Dis 2008;40 Suppl 2:S253-S259.

8. Loftus EV Jr, Harewood GC, Loftus CG, et al. PSC-IBD: a unique form of inflammatory bowel disease associated with primary sclerosing cholangitis. Gut 2005;54:91-96.

9. Pardi DS, Tremaine WJ, Sandborn WJ, McCarthy JT. Renal and urologic complications of inflammatory bowel disease. Am J Gastroenterol 1998;93:504-514.

10. Donadio JV, Grande JP. IgA nephropathy. N Engl J Med 2002; 347:738-748.

11. Takemura T, Okada M, Yagi K, Kuwajima H, Yanagida H. An adolescent with IgA nephropathy and Crohn disease: pathogenetic implications. Pediatr Nephrol 2002;17:863-866.

12. Ueno Y, Tanaka S, Onitake T, et al. Infliximab treatment for Crohn's disease in a patient with IgA nephropathy. Clin J Gastroenterol 2009;2:380-383.

13. Forshaw MJ, Guirguis O, Hennigan TW. IgA nephropathy in association with Crohn's disease. Int J Colorectal Dis 2005; 20:463-465.

14. Marek-Bukowiec K, Konieczny A, Ratajczyk K, Witkiewicz W. Candidate urine peptide biomarkers for IgA nephropathy: where are we now? Dis Markers 2018;2018:5205831.

15. O'Shaughnessy MM, Hogan SL, Thompson BD, Coppo R, Fogo AB, Jennette JC. Glomerular disease frequencies by race, sex and region: results from the International Kidney Biopsy Survey. Nephrol Dial Transplant 2018;33:661-669.

16. Satsangi J, Silverberg MS, Vermeire S, Colombel JF. The Montreal classification of inflammatory bowel disease: controversies, consensus, and implications. Gut 2006;55:749-753.

17. Rachmilewitz D. Coated mesalazine (5-aminosalicylic acid) versus sulphasalazine in the treatment of active ulcerative colitis: a randomized trial. BMJ 1989;298:82-86.

18. Best WR, Becktel JM, Singleton JW, Kern F Jr. Development of 
a Crohn's disease activity index. national cooperative Crohn's disease study. Gastroenterology 1976;70:439-444.

19. Utsunomiya Y, Koda T, Kado T, et al. Incidence of pediatric IgA nephropathy. Pediatr Nephrol 2003;18:511-515.

20. McGrogan A, Franssen CF, de Vries CS. The incidence of primary glomerulonephritis worldwide: a systematic review of the literature. Nephrol Dial Transplant 2011;26:414-430.

21. Ambruzs JM, Walker PD, Larsen CP. The histopathologic spectrum of kidney biopsies in patients with inflammatory bowel disease. Clin J Am Soc Nephrol 2014;9:265-270.

22. Wyatt RJ, Julian BA. IgA nephropathy. N Engl J Med 2013;368: 2402-2414.

23. Coppo R. The intestine-renal connection in IgA nephropathy. Nephrol Dial Transplant 2015;30:360-366.

24. Cesta MF. Normal structure, function, and histology of mucosa-associated lymphoid tissue. Toxicol Pathol 2006;34:599608.

25. Kiryluk K, Novak J. The genetics and immunobiology of IgA nephropathy. J Clin Invest 2014;124:2325-2332.

26. Nagy J, Scott H, Brandtzaeg P. Antibodies to dietary antigens in IgA nephropathy. Clin Nephrol 1988;29:275-279.

27. Kamata T, Nogaki F, Fagarasan S, et al. Increased frequency of surface IgA-positive plasma cells in the intestinal lamina propria and decreased IgA excretion in hyper IgA (HIGA) mice, a murine model of IgA nephropathy with hyperserum IgA. J Immunol 2000;165:1387-1394.

28. Kiryluk K, Novak J, Gharavi AG. Pathogenesis of immunoglobulin A nephropathy: recent insight from genetic studies. Annu Rev Med 2013;64:339-356.

29. Wehkamp J, Salzman NH, Porter E, et al. Reduced Paneth cell alpha-defensins in ileal Crohn's disease. Version 2. Proc Natl Acad Sci U S A 2005;102:18129-18134.

30. Hayashi R, Tsuchiya K, Fukushima K, et al. Reduced human a-defensin 6 in noninflamed jejunal tissue of patients with Crohn's disease. Inflamm Bowel Dis 2016;22:1119-1128. 